\title{
Anti-neuroinflammatory Activity of Nobiletin on Suppression of Microglial Activation
}

\author{
Yanji CuI,${ }^{a, e}$ Jinji Wu,${ }^{a, e}$ Sung-Cherl Jung, ${ }^{a, e}$ Deok-Bae PARK, ${ }^{a, f}$ Young-Hee MAeng, ${ }^{a, g}$ \\ Jeong Yun HonG, ${ }^{a, h}$ Se-Jae Kim, ${ }^{b}$ Sun-Ryung LeE, ${ }^{b}$ Soon-Jong Kim, ${ }^{c}$ \\ Sang Jeong KIM, ${ }^{d}$ and Su-Yong EuN*,a,e \\ ${ }^{a}$ Institute of Medical Science, Jeju National University School of Medicine; ${ }^{e}$ Department of Physiology, Jeju National \\ University School of Medicine; ${ }^{f}$ Department of Histology, Jeju National University School of Medicine; ${ }^{g}$ Department of \\ Pathology, Jeju National University School of Medicine; ${ }^{h}$ Department of Pediatrics, Jeju National University School of \\ Medicine; 66 Jejudaehakno, Jeju-si, Jeju-do 690-756, Republic of Korea: ${ }^{b}$ Department of Biology, Jeju National \\ University; 66 Jejudaehakno, Jeju-si, Jeju-do 690-756, Republic of Korea: ' Department of Chemistry, Mokpo National \\ University; 61 Dolim-Ri, Chonggye-Myon, Muan-Gun, Chonnam 534-729, Republic of Korea: and ${ }^{d}$ Department of \\ Physiology and Biophysics, Seoul National University College of Medicine; 28 Yonkeun-Dong, Chongno-ku, Seoul \\ 110-799, Republic of Korea. Received April 20, 2010; accepted August 27, 2010; published online August 30, 2010
}

A growing body of evidence suggests that nobiletin $\left(5,6,7,8,3^{\prime}, 4^{\prime}\right.$-hexamethoxy flavone) from the peel of citrus fruits, enhances the damaged cognitive function in disease animal models. However, the neuroprotective mechanism has not been clearly elucidated. Since nobiletin has shown anti-inflammatory effects in several tissues, we investigated whether nobiletin suppresses excessive microglial activation implicated in neurotoxicity in lipopolysaccharide (LPS)-stimulated BV-2 microglia cell culture models. Release of nitric oxide (NO), the major inflammatory mediator in microglia, was markedly suppressed in a dose-dependent manner following nobiletin treatment $(1-50 \mu \mathrm{M})$ in LPS-stimulated BV-2 microglia cells. The inhibitory effect of nobiletin was similar to that of minocycline, a well-known microglial inactivator. Nobiletin significantly inhibited the release of the proinflammatory cytokine tumor necrosis factor (TNF- $\alpha$ ) and interleukin-1 $\beta$ (IL-1 $\beta$ ). LPS-induced phosphorylations of extracellular signal-regulated kinase (ERK), c-Jun $\mathrm{NH}_{2}$-terminal kinase (JNK), and p38 mitogen-activated protein kinases (MAPKs) were also significantly inhibited by nobiletin treatment. In addition, nobiletin markedly inhibited the LPS-induced pro-inflammatory transcription factor nuclear factor $\kappa \mathrm{B}(\mathrm{NF}-\kappa \mathrm{B})$ signaling pathway by suppressing nuclear NF- $\kappa$ B translocation from the cytoplasm and subsequent expression of NF- $\kappa B$ in the nucleus. Taken together, these results may contribute to further exploration of the therapeutic potential and molecular mechanism of nobiletin in relation to neuroinflammation and neurodegenerative diseases.

Key words nobiletin; microglia; neuroinflammation; nuclear factor $\kappa \mathrm{B}$; neurodegeneration; citrus

Neuronal cell damage and death are attributed to the pathogenesis of neurodegenerative diseases and functional deterioration. Cognitive deficit shown in Alzheimer's disease (AD) is related to cell death of cholinergic neurons in basal forebrain, ${ }^{1,2)}$ dopaminergic neuronal cell death is related to Parkinson's disease (PD), ${ }^{3,4)}$ and motor neuronal cell death is related to amyotrophic lateral sclerosis (ALS). ${ }^{5)}$ The functional loss consequential to stroke could depend on the location and size of the ischemic area in the brain. ${ }^{6,7)}$

Microglial cells, the resident immune cells of the brain, exert a role of immune surveillance and host defense under physiological conditions. However, excessive microglial activation with overproduction of nitric oxide (NO) and proinflammatory cytokines, such as interleukin-1 $\beta$ (IL-1 $\beta$ ), interleukin-6 (IL-6) and tumor necrosis factor- $\alpha$ (TNF- $\alpha$ ) has been shown to be an important cause of neuronal cell death, and is implicated in neuroinflammation and neurodegeneration.

Microglial activation precedes neuronal cell death in several brain diseases including ischemia. ${ }^{8-10)}$ Therefore, microglia-mediated neurotoxicity is a crucial molecular mechanisms associated with neuronal cell death, damage, and functional deterioration. Suppression of microglial activation has been suggested as a potential therapeutic target to alleviate the progression of neuroinflammatory and neurodegenerative diseases. $^{11,12)}$

In the case of stroke, early blockade of microglial activa- tion could be a very effective treatment since it inhibits delayed neuronal cell death in the penumbra region of brain ischemia and as such could provide a therapeutic time window to alleviate further brain damage following initial neuronal cell death in an ischemic core area. ${ }^{13,14)}$ Microglial activation and subsequent neuroinflammation-mediated neurotoxicity is implicated as a target of therapeutic intervention not only in acute stroke injuries but also in chronic neurodegenerative diseases such as ischemia-reperfusion injury, PD, AD, and ALS. ${ }^{15-18)}$

Many compounds from natural resources have been investigated as candidates for drug development. The anti-inflammatory and anti-oxidative activities of citrus fruit peel extracts were evaluated in peripheral inflammatory cells, RAW 264.7 a murine macrophage cell line, and correlated with the content of nobiletin, a polymethoxylated flavones. ${ }^{19,20)}$ Nobiletin exerted the most potent inhibitory activity of NO in the RAW 264.7 cells among the tested flavonoids (naringin, naringenin, hesperidin, hesperetin, rutin, tangeretin and nobiletin) found in citrus fruit feels. ${ }^{20)}$

Recent evidence suggests that citrus nobiletin is active in the central nervous system. Citrus nobiletin has neurotrophic activity which induces neurites outgrowth by activating a cAMP response element (CRE) transcription-dependent signaling pathway in PC12D cells, ${ }^{21)}$ improving memory deterioration in MK-801-treated mice, olfactory bulbectomyinduced mice, ${ }^{22)}$ and a transgenic AD mouse model. ${ }^{23)} \mathrm{No}-$ 
biletin improved ischemia-induced learning and memory deficits through stimulation of calcium/calmodulin-dependent protein kinase II (CaMKII) and cyclic AMP-response element binding protein (CREB) phosphorylation. ${ }^{24)}$

The fact that nobiletin may suppress microglial activation has not yet been reported. Therefore, we investigated whether citrus nobiletin suppress microglial activation implicated in neuroinflammation and neurotoxicity in lipopolysaccharide (LPS)-stimulated BV-2 microglia. This study explores the therapeutic potential and molecular mechanism of nobiletin against neuroinflammation and neurodegeneration.

\section{MATERIALS AND METHODS}

Cell Culture BV-2 microglia, immortalized murine microglia cell line, exhibit phenotypic and functional properties comparable to those of primary microglia and hippocampal neurons. ${ }^{25,26)}$ BV-2 cells were obtained from Dr. E. Joe (Ajou University, Korea). Cells were cultured in Dulbecco's modified Eagle's medium (DMEM) containing 10\% fetal bovine serum (FBS) and kept at $37^{\circ} \mathrm{C}$ in a humidified atmosphere of $5 \% \mathrm{CO}_{2}$. The cells were seeded in 6-well plates $\left(1.2 \times 10^{6}\right.$ cells/well, Corning, U.S.A.) or $60 \mathrm{~mm}$ culture dishes $\left(3 \times 10^{6}\right.$ cells/well, Corning, U.S.A.).

Reagents Nobiletin, from the peel of Citrus sunki HorT. ex TANAKA, a nobiletin-rich citrus fruit, was isolated at the Jeju National University, Department of Biology. ${ }^{19)}$ Nobiletin purchased from Sigma was also used to compare activity. DMEM, FBS, penicillin, and streptomycin were obtained from Gibco BRL (U.S.A.). All other reagents were purchased from Sigma, unless indicated.

Lactate Dehydrogenase Release (LDH) Assay LDH is released into culture media upon damage to the cytoplasmic membrane, oxidizes lactate to pyruvate which reacts with iodonitrotetrazolium to form formazan (red). Therefore, the increase in the amount of formazan produced in culture media directly correlates with an increase in the number of lysed cells. The cytotoxicity of nobiletin was measured with an LDH cytotoxicity detection kit (Takara Shuzo Co., Japan) to determine the pharmacological dose for trial. BV-2 microglial cells were seeded on 96-well plates at $2 \times$ $10^{4}$ cells/well. Following a $12 \mathrm{~h}$ stabilization period, the cells were treated with different concentrations of nobiletin for $26 \mathrm{~h}$. A $50 \mu \mathrm{l}$ sample of supernatant was exposed to a $50 \mu \mathrm{l}$ LDH mixture and absorbance was read at $492 \mathrm{~nm}$ on a microplate reader. LDH release was then expressed as \% of maximal LDH release measured after lysis of the cells with $0.5 \%$ Triton X-100.

MTT Cell Viability Assay MTT [3-(4,5-dimethylthiazol-2-yl)-2,5-diphenyl tetrazolium bromide] was used to examine the effect of nobiletin on cell viability. MTT (yellow) is reduced to formazan (purple) by mitochondrial succinyl dehydrogenase in living cells. Therefore, the amount of formazan produced in cytoplasm is directly correlated with cell viability. BV-2 microglial cells were seeded on 24-well plates at $1 \times 10^{5}$ cells per well containing $500 \mu \mathrm{l}$ medium. After treatment, $250 \mu \mathrm{l}$ MTT $(2 \mathrm{mg} / \mathrm{ml})$ was added to each well and the mixture further incubated for $2 \mathrm{~h}$. The liquid in each well was then aspirated and $500 \mu \mathrm{l}$ dimethyl sulfoxide (DMSO) was added, mixed thoroughly on a shaker for $30 \mathrm{~min}$. Absorbance was subsequently read at $540 \mathrm{~nm}$ using a microplate reader (Model 550, Bio-Rad, U.S.A.)

NO Quantification The accumulation of $\mathrm{NO}_{2}^{-}$, a stable end product, extensively used as an indicator of $\mathrm{NO}$ production, was assayed using the Griess reagent. BV-2 microglial cells were seeded on 24-well tissue culture plates at $1 \times 10^{5}$ cells/well containing medium $(500 \mu \mathrm{l})$ and then incubated with LPS $(200 \mathrm{ng} / \mathrm{ml})$ for $24 \mathrm{~h}$ in the presence or absence of nobiletin. The supernatants were mixed with equal amounts of Griess reagent. Samples were incubated at room temperature for $10 \mathrm{~min}$ and absorbance was subsequently read at $540 \mathrm{~nm}$ using a microplate reader (Model 550, BioRad, U.S.A.).

Reverse Transcription-Polymerase Chain Reaction (RT-PCR) Total RNA was isolated using Tri reagent (Molecular Research Center Inc., U.S.A.) according to the manufacturer's instructions. Briefly, $1 \mu \mathrm{g}$ of total RNA was incubated in a tube containing $100 \mathrm{U}$ of SuperScript II Reverse Transcriptase (Invitrogen, U.S.A.), 100 U RNaseOUT (Invitrogen), $250 \mu \mathrm{M}$ deoxyribonucleotide triphosphate (dNTPs), and $0.5 \mu \mathrm{M}$ oligo $(\mathrm{dT})_{12-18}$ primer for $15 \mathrm{~min}$ at $70^{\circ} \mathrm{C}$. The reaction was terminated by heating the mixture at $70^{\circ} \mathrm{C}$ for 5 min followed by cooling on ice. The RNA strands in the RNA-DNA hybrid were removed by adding $10 \mathrm{U}$ RNaseH (Invitrogen, U.S.A.) and incubating for $45 \mathrm{~min}$ at $42^{\circ} \mathrm{C}$. Polymerase chain reaction (PCR) was performed in a total volume of $25 \mu \mathrm{l}$ containing $3 \mu \mathrm{l}$ cDNA, $200 \mu \mathrm{M}$ dNTPs, $20 \mathrm{pmol}$ of each primer pair, and $3 \mathrm{U}$ Taq DNA polymerase (iNtRON, U.S.A.) in Taq DNA polymerase reaction buffer as provided by the supplier. A total of 30 cycles were run under the following schemes; denaturing at $94{ }^{\circ} \mathrm{C}$ for $40 \mathrm{~s}$, primer annealing at $50^{\circ} \mathrm{C}(\mathrm{TNF}-\alpha)$ and $55^{\circ} \mathrm{C}$ glyceraldehyde-3-phosphate dehydrogenase (GAPDH), and an extension step at $72^{\circ} \mathrm{C}$ for $1 \mathrm{~min}$ in the Peltier Thermal Cycler (PTC)-100 (BIO-RAD, U.S.A.). The following sets of primers were used in the PCR amplifications: TNF- $\alpha$ (forward 5 '-ATGAGCACAGAAAGCATGAT-3', reverse 5'-TGACTTTCTCCTGGTATGA-3'), GAPDH (forward 5'-GTCCACATTGTTGCCATCAACGAC-3', reverse 5'-TTTCTCGTGGTTCACACCCATCAC-3'). The expected PCR products were $507 \mathrm{bp}$ for TNF- $\alpha$ and 336 bp for GAPDH, respectively. PCR products were separated electrophoretically on $1 \%$ agarose gel, stained with ethidium bromide, and subsequently visualized under UV illumination. Optical densities of the PCR bands for TNF- $\alpha$ were quantified with an Image J analyzer (http://rsb.info. nih.gov/ij/) and normalized with those of GAPDH.

Western Blotting Analysis Cells were washed once with phosphate-buffered saline (PBS; $\mathrm{pH} 7.4$ ) and lysed with modified radio-immunoprecipitation assay (RIPA) buffer (10 mM Tris- $\mathrm{HCl}$; pH 7.4, $150 \mathrm{~mm} \mathrm{NaCl,} 1 \mathrm{~mm}$ ethylene glycol bis(2-aminoethylether)- $N, N, N^{\prime}, N^{\prime}$-tetraacetic acid (EGTA), 0.1\% sodium dodecyl sulfate (SDS), $1 \mathrm{~mm} \mathrm{NaF,}$ $1 \mathrm{~mm} \mathrm{Na} \mathrm{VO}_{4}, 1 \mathrm{~mm}$ phenylmethylsulfonyl fluoride (PMSF), $1 \mu \mathrm{g} / \mathrm{ml}$ aprotinin, and $1 \mu \mathrm{g} / \mathrm{ml}$ leupeptin). Protein $(50 \mu \mathrm{g})$ was separated in $10 \%$ SDS-polyacrylamide gel and transferred to a nitrocellulose membrane using Towbin transfer buffer (192 mm glycine, $25 \mathrm{~mm}$ Tris, and 20\% methanol; $\mathrm{pH}$ $8.3)$. The blots were incubated with $5 \%$ skim milk in TTBS

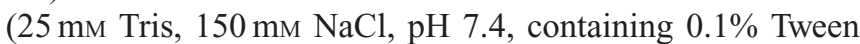
20) for $2 \mathrm{~h}$ at room temperature to block nonspecific binding. Subsequently, the membranes were probed with anti-extracellular signal-regulated kinase (ERK) and anti-phospho- 
ERK (1 : 1000, Santa Cruz Biotechnology Inc., U.S.A.), antip38 and anti-phospho-p38 (1:1000, Cell Signaling Laboratories, U.S.A.), anti-c-Jun $\mathrm{NH}_{2}$-terminal kinase (JNK) and anti-phospho JNK (1:1000, Cell Signaling Laboratories, U.S.A.), anti-inducible nitric oxide synthase (iNOS) (1:1000, Cell Signaling Laboratories, U.S.A.), anti-cyclooxygenase-2 (COX-2) (1 : 1000, Cell Signaling Laboratories, U.S.A.), nuclear factor $\kappa \mathrm{B}$ p65 (NF- $\kappa \mathrm{B}$ p65) $(1: 500$, Santa Cruz Biotechnology Inc., U.S.A.), anti-inhibitory $\kappa \mathrm{B}$ (I $\kappa \mathrm{B}) \alpha(1: 500$, Santa Cruz Biotechnology Inc., U.S.A.) and anti- $\beta$-actin antibody $(1: 5000$, Cell Signaling Laboratories, U.S.A.). The blots were washed 3 times with TTBS and incubated with horseradish peroxidase (HRP)-conjugated antimouse immunoglobulin $\mathrm{G}$ (IgG) antibody or anti-rabbit IgG secondary antibody (1:5000, Vector Laboratories, U.S.A.). After several washes, the blots were developed using an enhanced chemiluminescence reagent (Amersham Biosciences, U.S.A.) according to the manufacturer's instructions. Optical densities of the band were quantified with an Image J analyzer (http://rsb.info.nih.gov/ij/) and normalized with those of $\beta$-actin.

Cytoplasmic and Nuclear Protein Preparations Cells were harvested from culture dishes with trypsin and centrifuged at $12000 \times \boldsymbol{g}$ for $5 \mathrm{~min}$ at $4{ }^{\circ} \mathrm{C}$. Cell pellets were washed twice with ice-cold PBS and resuspended in ice-cold hypotonic buffer [10 mM Hepes ( $\mathrm{pH} 7.9$ ), $1.5 \mathrm{~mm} \mathrm{MgCl}_{2}$, $10 \mathrm{~mm} \mathrm{KCl}, 0.5 \mathrm{~mm}$ dithiothreitol (DTT), $10 \mu \mathrm{g} / \mathrm{ml}$ aprotinin, $0.2 \mathrm{~mm}$ PMSF]. Following incubation on ice for $10 \mathrm{~min}$, the cytoplasmic extract was obtained as supernatant by centrifuging for $5 \mathrm{~min}$ and then stored at $-80^{\circ} \mathrm{C}$ until use. $\mathrm{Nu}$ clear pellets were resuspended in ice-cold high-salt buffer [20 mm Hepes (pH 7.9), 20\% glycerol, $420 \mathrm{~mm} \mathrm{NaCl,} 1.5 \mathrm{~mm}$ $\mathrm{MgCl}_{2}, 0.2 \mathrm{~mm}$ EDTA, $0.5 \mathrm{~mm}$ DTT, and $0.2 \mathrm{~mm}$ PMSF] for $40 \mathrm{~min}$, and centrifuged for $10 \mathrm{~min}$. The supernatant nuclear protein extract was stored at $-80^{\circ} \mathrm{C}$ until use.

Enzyme Linked Immunosorbent Assay (ELISA) The amount of pro-inflammatory cytokines released into the culture medium were measured using mouse TNF- $\alpha$ and IL- $1 \beta$ ELISA kits based on the quantitative sandwich enzyme immunosorbent technique. The assay was performed according to the manufacturer's protocol (Invitrogen, U.S.A.). In brief, $50 \mu \mathrm{l}$ of each sample or standard and $50 \mu \mathrm{l}$ of biotinylated anti-mouse TNF- $\alpha$ and IL- $1 \beta$ antibody were incubated for 90 min on a 96 -well ELISA plate pre-coated with anti-mouse monoclonal TNF- $\alpha$ and IL- $\beta$ antibody. After 4 washes, horseradish peroxidase (HRP)-conjugated secondary antibody was incubated for $30 \mathrm{~min}$. After 4 additional washes, chromogen solution was incubated for $30 \mathrm{~min}$ in the dark after which the peroxidase-catalyzed color change was stopped by adding stop solution. The absorbance was read at a wavelength of $450 \mathrm{~nm}$ using a microplate reader (Model 550, Bio-Rad, U.S.A.).

Fluorescent Immunocytochemistry BV-2 microglial cells were seeded on $12 \mathrm{~mm}$-diameter cover glass $(0.3 \times$ $10^{5}$ cells/glass). After treatment, cells were fixed with $4 \%$ paraformaldehyde in PBS ( $\mathrm{pH} 7.4$ ) for 30 min and permeabilized in $0.5 \%$ triton X-100 in PBS for $15 \mathrm{~min}$. Nonspecific binding was blocked with 5\% normal goat serum in PBS for $30 \mathrm{~min}$. Subcellular localization of $\mathrm{NF}-\kappa \mathrm{B}$ was examined using rabbit polyclonal antibody against NF- $\kappa$ B p65 (1: 500, Santa Cruz Biotechnology Inc., U.S.A.) and Alexa Fluor 488-conjugated goat anti-rabbit antibody ( $1: 400$, Invitrogen, U.S.A.). Nuclear counterstaining was performed with Hoechst 33342 ( 1 : 2000, Invitrogen, U.S.A.). Prolong kit (Invitrogen, U.S.A.) was used to reduce fading. Cells were imaged on a confocal laser scanning microscope (FV500, Olympus Optical Co., Japan) with a cooled charge-coupled device camera, controlled by Flow View 4.2 software (Olympus Optical Co., Japan).

Statistical Analysis Data are expressed as mean value \pm standard error of mean (S.E.M.) of at least 3 independent experiments. Statistical analyses were performed using the $t$-test and one-way ANOVA. The differences between groups were considered to be statistically significant when $p<0.05$.

\section{RESULTS}

Cytotoxicity Test of Nobiletin Cytotoxicity of nobiletin was examined using a LDH release assay and MTT assay. Cells were treated for $26 \mathrm{~h}$ with different concentrations of nobiletin. Nobiletin did not show any cytotoxicity at concentrations less than $100 \mu \mathrm{M}$ (Fig. 1B); therefore, most experiments were accomplished at a concentration of $50 \mu \mathrm{M}$ of nobiletin on the basis of the cytotoxic dose information and dose-dependency of NO production (Fig. 2).

Inhibitory Effects of Nobiletin on iNOS Expression and NO Release in LPS-Stimulated BV-2 Microglia NO production has been the most widely used representative indicator of microglial activation among many inflammatory mediators. The iNOS expression was generated in activated mi-

A<smiles>COc1ccc(-c2cc(=O)c3c(OC)c(OC)c(OC)c(OC)c3o2)cc1OC</smiles>

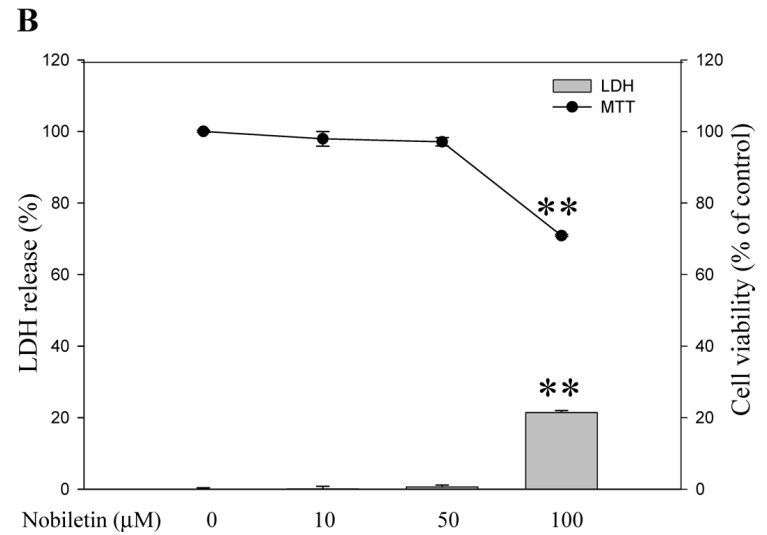

Fig. 1. (A) Chemical Structure of Nobiletin and (B) Cytotoxicity Test of Nobiletin in BV-2 Microglia

(A) Chemical skeletal structure of nobiletin, which is $5,6,7,8,3^{\prime}, 4^{\prime}$-hexamethoxyflavone, was shown. (B) Cells were treated with $26 \mathrm{~h}$ with different concentrations of nobiletin. Cytotoxicity of nobiletin was examined by LDH release assay. LDH release is expressed as \% of maximal LDH release measured after lysis of the cells with $0.5 \%$ Triton X-100. Values are the mean \pm S.E.M. of four samples in one independent experiment. The data were replicated in three repeated independent experiments. $* * p<0.001$ as compared to other concentrations. 
A

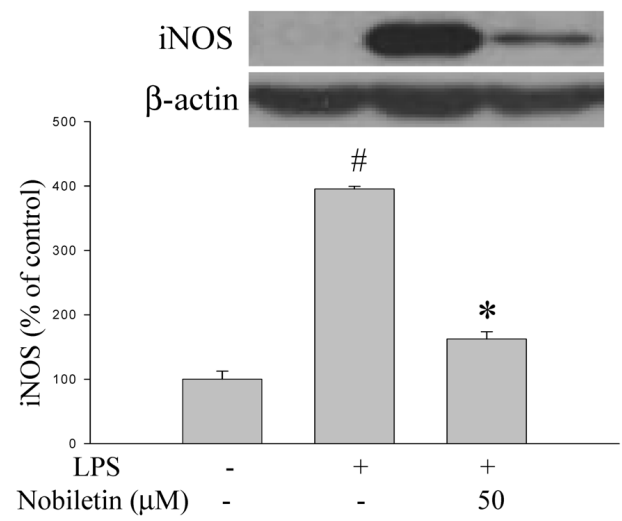

B

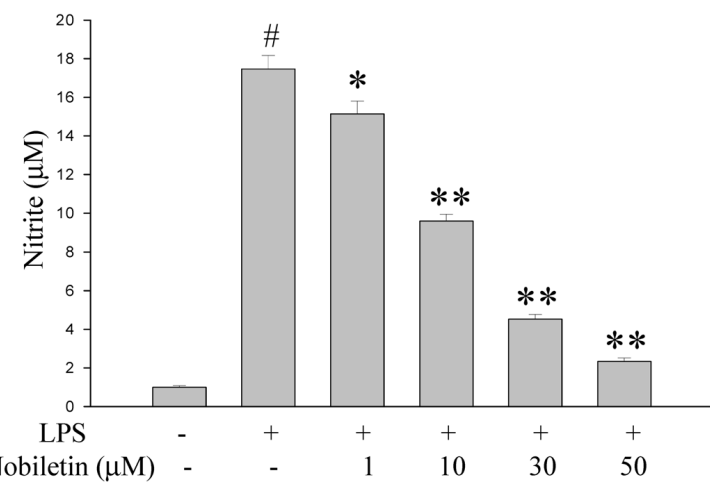

C

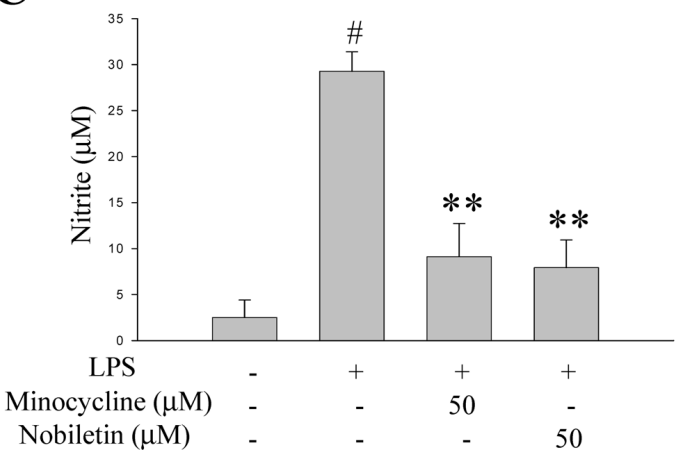

Fig. 2. Inhibitory Effects of Nobiletin on iNOS Expression and NO Release in LPS-Stimulated BV-2 Microglia

BV-2 microglial cells were treated with LPS $(100 \mathrm{ng} / \mathrm{ml})$ in the presence of absence of nobiletin $(50 \mu \mathrm{M})$ for $24 \mathrm{~h}$. Nobiletin was pretreated for $2 \mathrm{~h}$ before LPS stimulation. (A) Total cellular protein was isolated and LPS-induced iNOS expression levels were measured using Western blotting analysis. Optical densities of individual bands were normalized to the corresponding levels of $\beta$-actin. Figure shows the representative images from Western blot analysis and their quantitative analyses. (B) LPS-induced NO releases $(\mu \mathrm{M})$ were measured in culture medium at different concentrations using nitrite assay. (C) The inhibitory effects of nobiletin on NO release were compared to those of minocycline, a well-known microglia inactivator. Values are the mean \pm S.E.M. of four samples in one independent experiment. The data were replicated in three repeated independent experiments. $\# p<0.001$ as compared to untreated control group and $* p<0.05 ; * * p<0.001$ as compared to LPS alone-treated group.

croglial cells, mediating synthesis of NO, which were then released into culture medium.

The inhibitory effect of nobiletin on iNOS expression and NO release in LPS-stimulated BV-2 microglia was evaluated using Western blotting analysis and a nitrite assay (Fig. 2). BV-2 microglial cells were treated with LPS $(100 \mathrm{ng} / \mathrm{ml})$ in the presence or absence of nobiletin for $24 \mathrm{~h}$. A $2 \mathrm{~h}$ pretreatment with nobiletin was carried out prior to LPS stimulation. Nobiletin treatment $(50 \mu \mathrm{M})$ significantly inhibited LPS-induced iNOS expression by $71.2 \pm 10.7 \%$, when compared to the LPS alone-treated group (Fig. 2A). Nobiletin treatment
$(50 \mu \mathrm{M})$ also significantly inhibited protein expression of proinflammatory enzyme cyclooxygenase- 2 (COX-2) by $46.0 \pm 6.7 \%$ in LPS-stimulated microglia (data not shown). Nobiletin treatment $(1,10,30,50 \mu \mathrm{M})$ markedly suppressed LPS-induced NO release in a dose-dependent manner by $13.3 \pm 4.0 \%, 45.1 \pm 3.4 \%, 74.1 \pm 4.9 \%$ and $86.6 \pm 7.2 \%$ respectively (Fig. 2B). The inhibitory effect of nobiletin was similar to that of minocycline, a well-known microglia inactivator $^{13)}$ (Fig. 2C).

Inhibitory Effects of Nobiletin on TNF- $\alpha$ and IL-1 $\beta$ Production in LPS-Stimulated BV-2 Microglia Proinflammatory cytokines are soluble mediators of inter- and intra-cellular communication which occurs during inflammatory responses. The effect of nobiletin on proinflammatory cytokine release was investigated in LPS-stimulated BV-2 microglial cells treated with LPS $(100 \mathrm{ng} / \mathrm{ml})$ in the presence or absence of nobiletin $(50 \mu \mathrm{M})$ for $24 \mathrm{~h}$. Cells were pretreated with nobiletin for $2 \mathrm{~h}$ prior to LPS stimulation. RT/PCR, Western blotting, and ELISA analyses demonstrated that TNF- $\alpha$ mRNA expression (Fig. 3A), TNF- $\alpha$ protein expression (Fig. 3B) and TNF- $\alpha$ release into culture medium (Fig. 3C) were markedly increased 525.4 $23.5 \%$, $395.4 \pm 4.3 \%$ and $872.0 \pm 6.4 \%$ in the LPS-stimulated microglia group compared to unstimulated control group, and significantly suppressed in the nobiletin-treated group by $52.5 \pm 2.3 \%, 59.0 \pm 11.6 \%$ and $46.3 \pm 8.6 \%$ compared to the LPS alone-treated group. IL- $1 \beta$ release (Fig. 3D) were also increased $255.1 \pm 1.8 \%$ in the LPS-stimulated group and significantly suppressed by $14.9 \pm 0.9 \%$ in the nobiletin-treated group.

Inhibitory Effects of Nobiletin on Phosphorylation of ERK, JNK and p38 MAPKs in LPS-Stimulated BV-2 Microglia Following a $24 \mathrm{~h}$ nobiletin pretreatment, BV-2 microglial cells were treated with LPS $(100 \mathrm{ng} / \mathrm{ml})$ in the presence or absence of nobiletin $(50 \mu \mathrm{M})$ for $30 \mathrm{~min}$. After LPS stimulation, phosphorylations of mitogen-activated protein kinases (MAPKs) including ERK, JNK and p38 were investigated to determine whether they were involved in nobiletin's mechanism of action. Cell lysates were prepared and subjected to $10 \%$ SDS-PAGE followed by Western blotting analysis with antibodies specific for phosphorylated forms of ERK, JNK and p38 MAPK.

Phosphorylations of ERK (Fig. 4B), p38 (Fig. 4C) and JNK (Fig. 4D) were markedly increased in the LPS-stimulated microglia group $159.8 \pm 9.5 \%, 297.2 \pm 8.3 \%$, and $169.5 \pm 4.2 \%$ respectively, compared to the control microglia group without LPS treatment. However, nobiletin treatment significantly attenuated the phosphorylation levels of ERK, p38, and JNK by $30.9 \pm 3.6 \%, 37.0 \pm 7.0 \%$, and $21.2 \pm 2.0 \%$ respectively, compared to the LPS alone-treated group (Fig 4).

Upon activation, microglia show hypertrophic morpholological changes, increased motility, and the capacity to release inflammatory mediators such as NO and proinflammatory cytokines. ${ }^{27)}$ Since LPS-induced activation of MAPK such as ERK, p38, and JNK may be involved early in initiating the microglial activation processes, the suppressive effect of LPS-induced MAPK phosphorylation may be important in evaluating the effect of nobiletin on inhibiting microglial activation.

Inhibitory Effects of Nobiletin on NF-אB Signaling in 
A
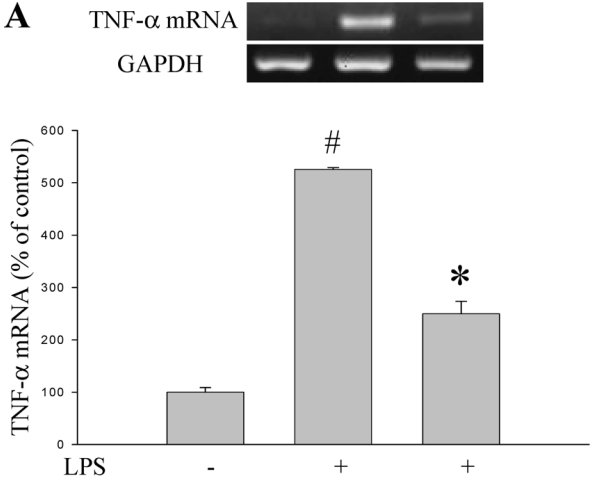

Nobiletin $(\mu \mathrm{M})$

C

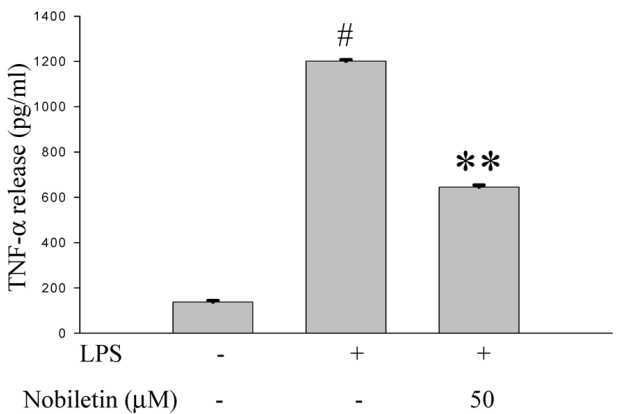

B
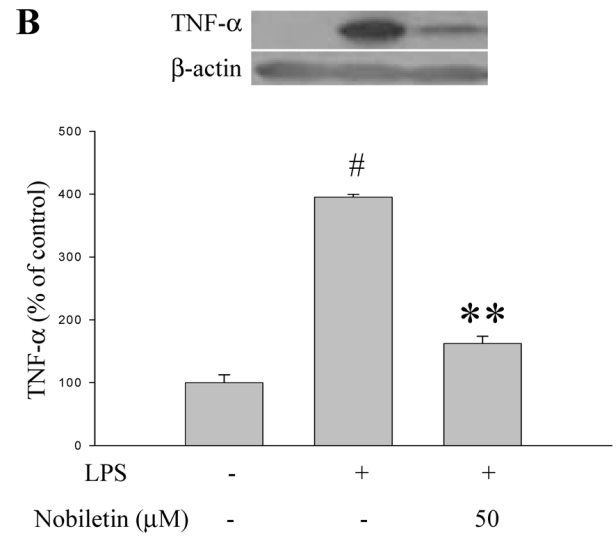

D

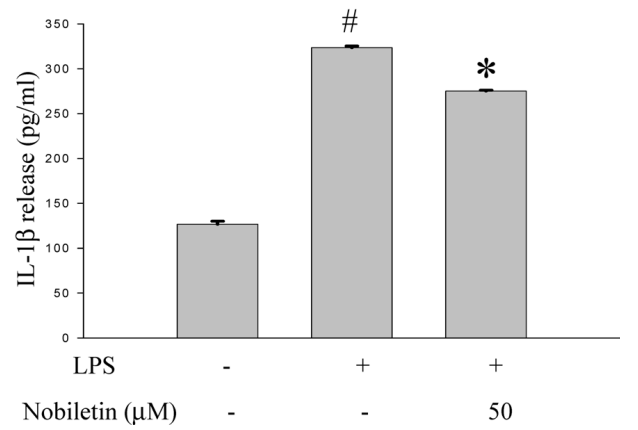

Fig. 3. Inhibitory Effects of Nobiletin on TNF- $\alpha$ and IL-1 $\beta$ Production in LPS-Stimulated BV-2 Microglia

BV-2 microglial cells were treated with LPS $(100 \mathrm{ng} / \mathrm{ml})$ for in the presence of absence of nobiletin $(50 \mu \mathrm{M})$ for $24 \mathrm{~h}$. Nobiletin was pretreated for $2 \mathrm{~h}$ before LPS stimulation. (A, B) Total cellular mRNA and protein were isolated and optical densities of individual TNF- $\alpha$ mRNA and protein bands were normalized to the corresponding levels of GAPDH mRNA and $\beta$-actin. Figure shows the representative images from RT-PCR and Western blotting analyses. Amounts of TNF- $\alpha$ (C) and IL-1 $\beta$ (D) released into the culture medium were measured using TNF- $\alpha$ and IL- $1 \beta$ ELISA kits based on the quantitative sandwich enzyme immunosorbent technique. Quantitative analyses were shown as $\%$ or fold increases of control unstimulated group. Values are the mean \pm S.E.M. of four samples in one independent experiment. The data were replicated in three repeated independent experiments. $\# p<0.001$ as compared to untreated control group and $* p<0.05 ; * * p<0.001$ as compared to LPS alone-treated group.

LPS-Stimulated BV-2 Microglia The signaling pathways of the major proinflammatory transcription factor NF- $\kappa \mathrm{B}$ are well established. ${ }^{19)}$ Briefly, NF- $\kappa \mathrm{B}$ is localized in the cytoplasm and bound to the inhibitory protein $\mathrm{I} \kappa \mathrm{B}$ in resting microglia. Microglia activation evokes I $\kappa$ B kinase (IKK) complex-induced phosphorylation of $\mathrm{I} \kappa \mathrm{B}$, which leads to its degradation and subsequent translocation of NF- $\kappa \mathrm{B}$ to the nucleus. The nuclear NF- $\kappa \mathrm{B}$ activates the transcription of target genes which have NF- $\kappa \mathrm{B}$-binding sites, such as TNF$\alpha$, IL- $1 \beta$ and iNOS. ${ }^{11,12)}$ Therefore, we analyzed whether nobiletin affects LPS-induced $\mathrm{I} \kappa \mathrm{B}$ degradation and NF- $\kappa \mathrm{B}$ translocation to the nucleus and subsequent nuclear NF- $\kappa \mathrm{B}$ expression levels.

Following $24 \mathrm{~h}$ of nobiletin pretreatment, BV-2 microglial cells were treated with LPS $(100 \mathrm{ng} / \mathrm{ml})$ in the presence or absence of nobiletin for the indicated times (15, $30 \mathrm{~min}, 1$, $2 \mathrm{~h})$. After LPS stimulation, total cellular protein was isolated and subjected to $10 \%$ SDS-PAGE followed by Western blotting analysis with antibodies specific for $\mathrm{I} \kappa \mathrm{B} \alpha$. In addition, nuclear protein fraction was also isolated (see Materials and Methods) to investigate NF- $\kappa \mathrm{B}$ expression in the nucleus.

$\mathrm{I} \kappa \mathrm{B} \alpha$ levels were markedly decreased with LPS treatment by $21.4 \pm 1.4 \%$ at the $15 \mathrm{~min}$ time point and maximally decreased by $56.7 \pm 13.7 \%$ at the $30 \mathrm{~min}$ time point compared to the unstimulated control group, suggesting LPS-induced $\mathrm{I} \kappa \mathrm{B}$ degradation. The reduced $\mathrm{I} \kappa \mathrm{B} \alpha$ levels recovered to $69.6 \pm 12.7 \%$ at the $1 \mathrm{~h}$ time point and $79.2 \pm 6.0 \%$ at the $2 \mathrm{~h}$ time point, compared to the unstimulated control group (Fig. $5 \mathrm{~A})$. Nobiletin treatment did not affect $\mathrm{I} \kappa \mathrm{B} \alpha$ levels in our experiment (Fig. 5B). Western blotting analysis with nuclear protein fraction demonstrated that NF- $\kappa \mathrm{B}$ p 65 subunit levels were dramatically increased $188.5 \pm 5.7 \%$ in the nuclei of the BV-2 microglia group stimulated with LPS for $30 \mathrm{~min}$ and nobiletin treatment significantly suppressed LPS-induced $\mathrm{NF}-\kappa \mathrm{B}$ p65 subunit expression in the nucleus by $29.0 \pm 8.5 \%$ compared to the LPS alone-treated group (Fig. 5C).

Fluorescent immunocytochemistry data visually demonstrated AF488 (green)-labeled NF- $\kappa$ B p65 subunit immunoreactivities shown in cytoplasms peripheral to the nuclei counterstained with Hoechst 33342 (blue) in the control microglia group (Figs. 5D-1-3). In the BV-2 microglia group stimulated with LPS for $30 \mathrm{~min}, \mathrm{NF}-\kappa \mathrm{B}$ p 65 subunit immunoreactivities were shown in the nuclei and barely found in the cytoplasms, suggesting translocation of NF- $\kappa \mathrm{B}$ to the nucleus (Figs. 5D-4-6). Nobiletin treatment markedly reversed nuclear translocation of the LPS-induced NF- $\kappa \mathrm{B}$ p65 subunit (Figs. 5D-7-9).

\section{DISCUSSION}

We investigated whether nobiletin suppresses brain insultinduced excessive microglial activation implicated in neuroinflammation and neurotoxicity. The results demonstrate that nobiletin markedly suppressed microglial activation 
A

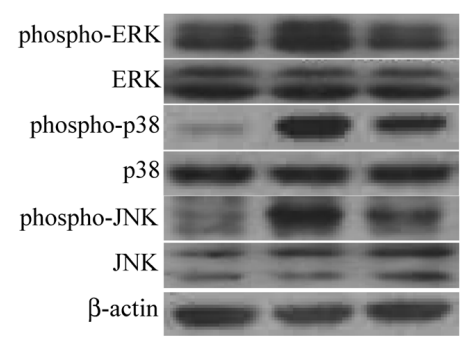

C

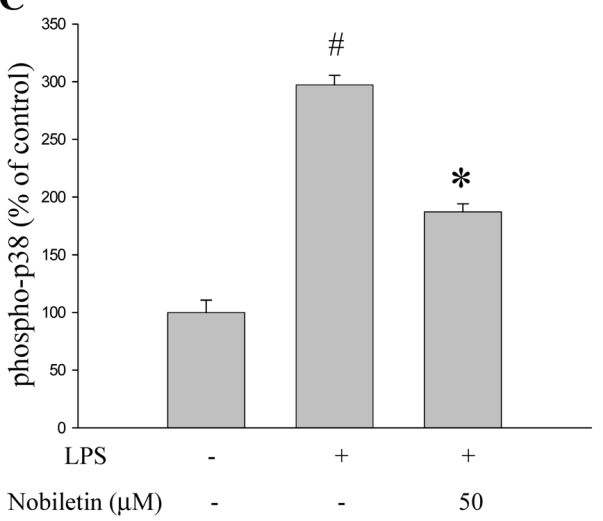

B

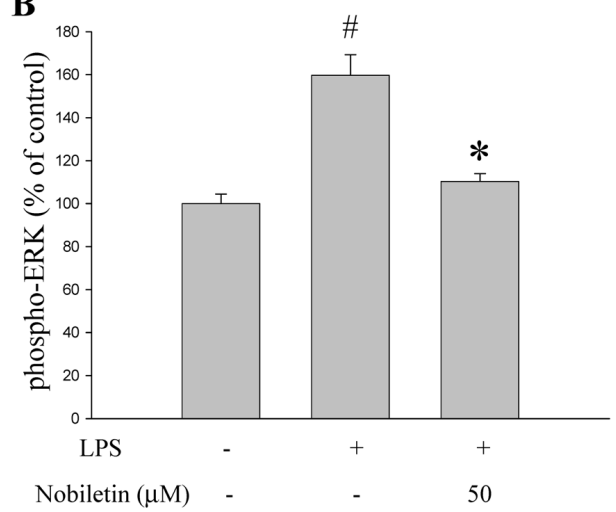

D

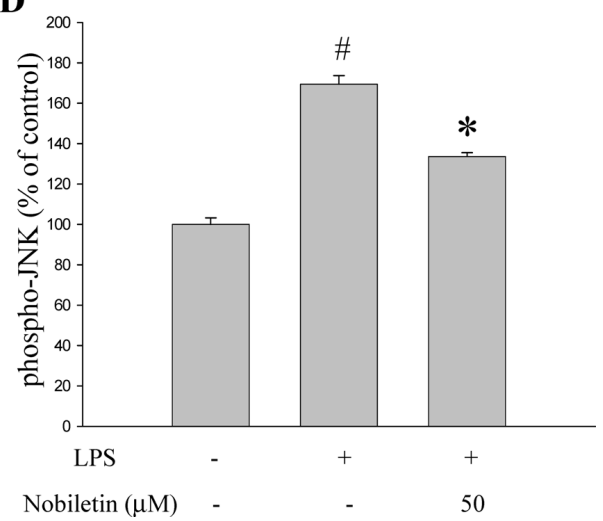

Fig. 4. Inhibitory Effects of Nobiletin on Phosphorylation of ERK, JNK and p38 MAPK in LPS-Stimulated BV-2 Microglia

BV-2 microglial cells were treated with LPS $(100 \mathrm{ng} / \mathrm{ml})$ for in the presence of absence of nobiletin ( $50 \mu \mathrm{M})$ for 30 min. Nobiletin was pretreated for $24 \mathrm{~h}$ before LPS stimulation. Cell lysates were then prepared and subjected to 10\% SDS-PAGE followed by Western blotting with antibodies specific for phosphorylated forms of ERK, JNK and p38 MAPK. Figure shows the representative images of protein bands (A) and their quantitative analyses (B-D). Optical densities of individual protein bands were normalized to the corresponding levels of $\beta$-actin. Groups were compared by $\%$ of control unstimulated group. Values are the mean \pm S.E.M. of four samples in one independent experiment. The data were replicated in three repeated independent experiments. $\# p<0.001$ as compared to untreated control group and $* p<0.05$ as compared to LPS alone-treated group.

characterized by overproduction and release of NO (Fig. 2) and pro-inflammatory cytokine TNF-alpha (Fig. 3). Activation of ERK, JNK, p38 MAPKs (Fig. 4), and NF- $\kappa$ B signaling pathways (Fig. 5) were significantly inhibited by nobiletin treatment in LPS-stimulated BV-2 microglial cells.

Consistent with previous reports on the signal pathways underlying microglial activation, ${ }^{11,12}$ we observed that MAPKs such as ERK, JNK and p38, NF- $\kappa$ B signal pathways and proinflammatory mediator $\mathrm{NO}$ and $\mathrm{TNF}-\alpha$ were involved in LPS-stimulated BV-2 microglial activation. We found all of these signal pathways to be significantly suppressed in nobiletin-treated BV-2 microglial cells, suggesting novel anti-neuroinflammatory activity of nobiletin on suppression of LPS-induced microglial activation. BV-2 microglia exhibit phenotypic and functional properties comparable to those of primary microglia. The experimental results shown in primary microglia culture are almost same to those accomplished in BV-2 microglia in many studies ${ }^{28,29)}$ including our previous investigation. ${ }^{30}$

Microglial cells have been shown to be activated by several endogenous and exogenous stimuli, including LPS, ${ }^{29}$ glutamate, ${ }^{30)}$ thrombin, ${ }^{31,32}$ ganglioside, ${ }^{33)}$ and beta amyloid. ${ }^{34)}$ LPS, a type of cell wall compound toxin of gram-negative bacteria, is not a common cause of human neuroinflammation; however, the experimental model of LPS stimulation has been the most widely used because it has shown potent capacity to stimulate microglia and is the most established among the known microglial activators.

Overproduction of NO and pro-inflammatory cytokines such as TNF- $\alpha$, IL- $1 \beta$ and IL- 6 released by activated microglia induces neuronal death and damage to neighboring neurons in the brain. The microglia-mediated neurotoxicity has been demonstrated in many investigations including a mouse model of neuroinflammation using intraperitoneally injection of LPS, ${ }^{12}$ and in vitro studies using a neuron-microglia co-culture model or a microglia-conditioned media method. ${ }^{11,29,35)}$ Therefore, microglial activation and subsequent neuroinflammation-mediated neurotoxicity is implicated as a target of therapeutic intervention not only in acute stroke injuries but also in chronic neurodegenerative diseases.

Inflammation exerts two opposite impacts in tissues and cells. Inflammation is basically a physiological response to recover damaged tissue and plays a role in innate immunity. However, inflammation turns into a pathophysiological condition which evokes diseases if it exceeds the cytotoxicity threshold. The initial causes evoking specific diseases are thought to be different in neuroinflammatory and neurodegenerative diseases such as ischemia, Parkinson's, Alzheimer's and ALS. However, neuroinflammation consequent to the initial causes (i.e., accumulation of beta amyloid, blockade of blood supply, etc.), shows common features regardless of disease entity. It is now generally accepted that neuroinflammation is a crucial factor actively involved in the 
A

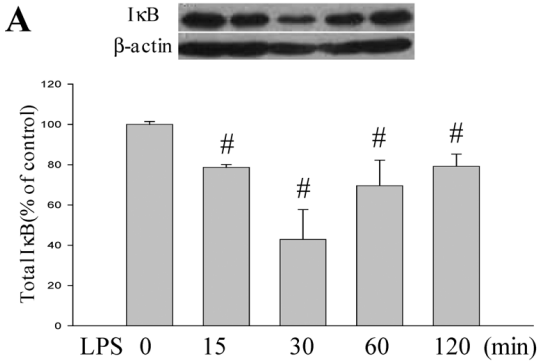

B

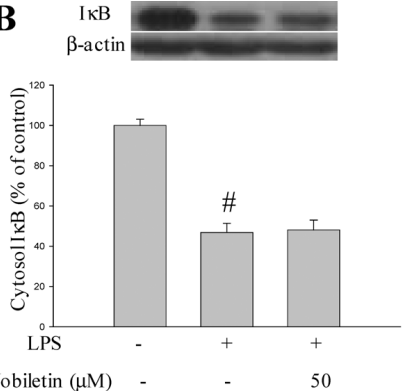

C
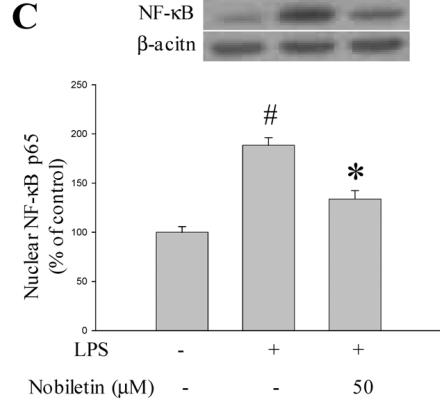

D

NF- $\kappa \mathrm{B}$

Hoechst 33342

Merge

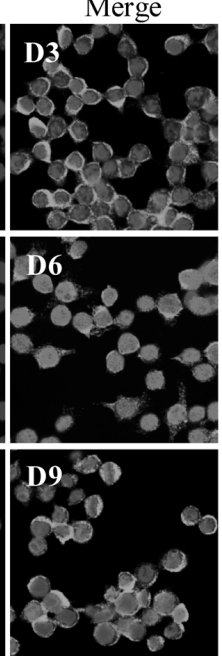

Fig. 5. Inhibitory Effects of Nobiletin on NF- $\kappa$ B Signaling in LPS-Stimulated BV-2 Microglia

BV-2 microglial cells were treated with LPS $(100 \mathrm{ng} / \mathrm{ml})$ in the presence or absence of nobiletin $(50 \mu \mathrm{M})$ for the indicated time $(15,30 \mathrm{~min}, 1,2 \mathrm{~h})$. Nobiletin was pretreated for $24 \mathrm{~h}$ before LPS stimulation. (A, B) Total cellular protein was isolated and $\mathrm{I} \kappa \mathrm{B} \alpha$ protein levels were measured to investigate LPS-induced I $\kappa \mathrm{B}$ degradation using Western blotting analysis. (C) After LPS stimulation ( $30 \mathrm{~min})$, nuclear protein was isolated from total cellular protein and NF- $\kappa$ B p65 protein levels were measured using Western blotting analysis to investigate LPS-induced NF- $\kappa$ B expression in the nucleus. Optical densities of individual protein bands were normalized to the corresponding levels of $\beta$-actin. Figure shows the representative images of protein bands and their quantitative analyses. Groups were compared by $\%$ of control unstimulated group. Values are the mean \pm S.E.M. of four samples in one independent experiment. The data were replicated in three repeated independent experiments. $\# p<0.001$ as compared to untreated control group and $* p<0.05$ as compared to LPS alone-treated group. (D) Representative images of fluorescent immunocytochemistry were shown at 200-fold magnification to demonstrate effects of nobiletin on LPS-induced alteration of NF- $\kappa \mathrm{B}$ subcellular localization. AF488 (green)-labeled NF- $\kappa \mathrm{B}$ p65 subunit immunoreactivities were shown in the cytoplasms peripheral to nuclei counterstained with Hoechst 33342 (blue) in control microglia group (D-1-3). In LPS-treated microglia group, NF- $\kappa$ B p65 subunit immunoreactivities were shown in nuclei (Figs. D-4 - 6). Nobiletin treatment markedly reversed LPS-induced NF- $\kappa$ B p65 subunit translocation (D-7-9).

initiation and progress of diseases.

Neuroinflammation, which is mainly mediated by activation of microglia, is able to deteriorate neural function via synaptic loss and dysfunction several years prior to severe neuronal death and damage. ${ }^{36,37)}$ Inflammation decreased the number of $N$-methyl-D-aspartate receptors (NMDAR) within the dentate gyrus and CA3 hippocampal areas. ${ }^{38)}$ Inflammation impaired spatial memory and induction of synaptic plasticity including both long term potentiation (LTP) and depression (LTD) in perforant path-granule cell synapses. ${ }^{37,39)}$ Several epidemiological cohort studies have demonstrated the reduced risk and delayed onset of Alzheimer's disease in non-steroidal anti-inflammatory drug (NSAID) users, ${ }^{40-42)}$ suggesting a neuroprotective effect of anti-inflammatory agents in the pathogenesis and progression of neurodegenerative diseases. Therefore, the suppression of microglial activation may be an effective therapeutic strategy to alleviate the progression of neuroinflammatory and neurodegenerative diseases.

Citrus fruit-derived flavonoids and their metabolites have significant biological activities, including anti-inflammatory properties. The dried peels have been used as herbal medicines to improve bronchial asthma, dyspepsia and cardiac circulation. ${ }^{20)}$ Nobiletin, a polymethoxylated flavone exclusively found in the fruit peel of Citrus, is the most potent among tested citrus flavonoids in inhibitory activity of NO production in stimulated RAW 264.7 cells, a macrophage cell line. ${ }^{20)}$

Recently, a growing body of evidence has demonstrated the biological activity of nobiletin in the central nervous system. Nobiletin enhanced neurite outgrowth in PC12D cells by activating ERK/MAP kinase-dependent signaling to increase CREB phosphorylation. ${ }^{21)}$ Nobiletin enhanced PKAmediated phosphorylation of AMPA receptor subunit GluR1 and postsynaptically augmented field excitatory postsynaptic potential (fEPSP) in hippocampal slice related to cellular mechanisms of learning and memory. ${ }^{43)}$ Nobiletin rescued impaired memory in olfactory-bulbectomized mice accompanied by cholinergic neurodegeneration and reversed olfactory bulbectomy-induced reduction of acetylcholinesterase and choline acetyltransferase. ${ }^{22)}$

Nobiletin improved learning and memory impairment in several brain disease models, such as a $\mathrm{N}$-ethyl-D-aspartate receptor antagonism model, ${ }^{44)}$ an ischemia animal model with bilateral common carotid artery occlusion (BCCAO) ${ }^{24)}$ and an amyloid precursor protein (APP) transgenic mouse 
model which over expresses human APP 695 harboring the double Swedish and London mutation. ${ }^{23)}$ The effects of nobiletin on cognitive functions shown in these brain disease models were related to phosphorylation of ERK/MAP kinase, CREB and CaMKII.

In conclusion, we demonstrated that citrus nobiletin suppresses microglial activation in LPS-stimulated BV-2 microglial cell culture model. Even though several studies have been tried to explore the neuroprotective mechanism of nobiletin, it remains to be fully elucidated. Anti-neuroinflammatory activity of nobiletin on suppression of brain insultinduced excessive microglial activation was suggested as one of the neuroprotective mechanisms in the present study. These results may contribute to further exploration of the therapeutic potential and molecular mechanism of nobiletin in relation to neuroinflammation and neurodegenerative diseases.

Acknowledgments This work was supported by the research grant of Jeju National University in 2007.

\section{REFERENCES}

1) Christensen D. Z., Bayer T. A., Wirths O., Neurobiol. Aging, 31 1153-1163 (2008).

2) Wu C. K., Thal L., Pizzo D., Hansen L., Masliah E., Geula C., Exp. Neurol., 195, 484-496(2005)

3) Caudle W. M., Zhang J., Exp. Neurol., 220, 230-233 (2009).

4) Lees A. J., Hardy J., Revesz T., Lancet, 373, 2055-2066 (2009).

5) Van Damme P., Robberecht W., Curr. Opin. Neurol., 22, 486-492 (2009).

6) Ochfeld E., Newhart M., Molitoris J., Leigh R., Cloutman L., Davis C., Crinion J., Hillis A. E., Stroke, 41, 325-330 (2010).

7) Wang Y. D., Li X. P., Li M., Liu S., Lu X. P., Peng Y., Huang R. X. Eur. Neurol., 63, 52-59 (2009).

8) Cho I. H., Hong J., Suh E. C., Kim J. H., Lee H., Lee J. E., Lee S., Kim C. H., Kim D. W., Jo E. K., Lee K. E., Karin M., Lee S. J., Brain, 131, 3019-3033 (2008).

9) Rupalla K., Allegrini P. R., Sauer D., Wiessner C., Acta Neuropathol., 96, 172-178 (1998).

10) Zhang Z., Chopp M., Powers C., Brain Res., 744, 189-198 (1997).

11) Park G. H., Jeon S. J., Ko H. M., Ryu J. R., Lee J. M., Kim H. Y., Han S. H., Kang Y. S., Park S. H., Shin C. Y., Ko K. H., Nitric Oxide, 22 $18-29$ (2010).

12) Ock J., Kim S., Yi K. Y., Kim N. J., Han H. S., Cho J. Y., Suk K., Biochem. Pharmacol., 79, 596-609 (2010).

13) Tikka T., Fiebich B. L., Goldsteins G., Keinanen R., Koistinaho J., J. Neurosci., 21, 2580-2588 (2001).

14) Yrjänheikki J., Tikka T., Keinänen R., Goldsteins G., Chan P. H., Koistinaho J., Proc. Natl. Acad. Sci. U.S.A., 96, 13496-13500 (1999).

15) Breitner J. C., Gau B. A., Welsh K. A., Plassman B. L., McDonald W. M., Helms M. J., Anthony J. C., Neurology, 44, 227-232 (1994).

16) Combs C. K., Johnson D. E., Karlo J. C., Cannady S. B., Landreth G. E., J. Neurosci., 20, 558-567 (2000).

17) Kim H. S., Suh Y. H., Behav. Brain Res., 196, 168-179 (2009).

18) Lu L., Li F., Wang X., CNS Neurol. Disord. Drug Targets, 9, 232-240 (2009)
19) Choi S. Y., Hwang J. H., Ko H. C., Park J. G., Kim S. J., J. Ethnopharmacol., 113, 149-155 (2007).

20) Choi S. Y., Ko H. C., Ko S. Y., Hwang J. H., Park J. G., Kang S. H., Han S. H., Yun S. H., Kim S. J., Biol. Pharm. Bull., 30, 772-778 (2007).

21) Nagase H., Omae N., Omori A., Nakagawasa O., Tadano T., Yokosuka A., Sashida Y., Mimaki Y., Yamakuni T., Ohizumi Y., Biochem. Biophys. Res. Commun., 337, 1330-1336 (2005).

22) Nakajima A., Yamakuni T., Haraguchi M., Omae N., Song S. Y., Kato C., Nakagawasai O., Tadano T., Yokosuka A., Mimaki Y., Sashida Y., Ohizumi Y., J. Pharmacol. Sci., 105, 122-126 (2007).

23) Onozuka H., Nakajima A., Matsuzaki K., Shin R. W., Ogino K., Saigusa D., Tetsu N., Yokosuka A., Sashida Y., Mimaki Y., Yamakuni T., Ohizumi Y., J. Pharmacol. Exp. Ther, 326, 739-744 (2008).

24) Yamamoto Y., Shioda N., Han F., Moriguchi S., Nakajima A., Yokosuka A., Mimaki Y., Sashida Y., Yamakuni T., Ohizumi Y., Fukunaga K., Brain Res., 1295, 218 -229 (2009).

25) Blasi E., Barluzzi R., Bocchini V., Mazzolla R., Bistoni F., J. Neuroimmunol., 27, 229-237 (1990).

26) Bocchini V., Mazzolla R., Barluzzi R., Blasi E., Sick P., Kettenmann H., J. Neurosci. Res., 31, 616-621 (1992)

27) Eun S. Y., Kim E. H., Kang K. S., Kim H. J., Jo S. A., Kim S. J., Jo S. H., Kim S. J., Blackshear P. J., Kim J., Exp. Mol. Med., 38, 310-319 (2006).

28) Min K. J., Pyo H. K., Yang M. S., Ji K. A., Jou I., Joe E. H., Glia, 48 $197-206$ (2004)

29) Ock J., Kim S., Suk K., Pharmacol. Res., 59, 414-422 (2009).

30) Eun S. Y., Hong Y. H., Kim E. H., Jeon H., Suh Y. H., Lee J. E., Jo C. Jo S. A., Kim J., Biochem. Biophys. Res. Commun., 325, 320-327 (2004).

31) Choi S. H., Joe E. H., Kim S. U., Jin B. K., J. Neurosci., 23, 58775886 (2003).

32) Ryu J., Pyo H., Jou I., Joe E., J. Biol. Chem., 275, 29955-29959 (2000).

33) Pyo H., Joe E., Jung S., Lee S. H., Jou I., J. Biol. Chem., 274, 34584 34589 (1999)

34) Combs C. K., Karlo J. C., Kao S. C., J. Neurosci., 21, 1179-1188 (2001).

35) Kaushal V., Schlichter L. C., J. Neurosci., 28, 2221-2230 (2008).

36) Parameshwaran K., Dhanasekaran M., Suppiramaniam V., Exp. Neurol., 210, 7-13 (2008).

37) Min S. S., Quan H. Y., Ma J., Han J. S., Jeon B. H., Seol G. H., Neurosci. Lett., 456, 20-24 (2009).

38) Rosi S., Ramirez-Amaya V., Hauss-Wegrzyniak B., Wenk G. L., J. Neuroinflammation, 1, 12 (2004).

39) Min S. S., Quan H. Y., Ma J., Lee K. H., Back S. K., Na H. S., Han S H., Yee J. Y., Kim C., Han J. S., Seol G. H., Biochem. Biophys. Res. Commun., 383, 93-97 (2009).

40) Breitner J. C., Haneuse S. J., Walker R., Dublin S., Crane P. K., Gray S. L., Larson E. B., Neurology, 72, 1899-1905 (2009).

41) in t' Veld B. A., Ruitenberg A., Hofman A., Launer L. J., van Duijn C. M., Stijnen T., Breteler M. M., Stricker B. H., N. Engl. J. Med., 345, 1515-1521 (2001)

42) Szekely C. A., Thorne J. E., Zandi P. P., Ek M., Messias E., Breitner J C., Goodman S. N., Neuroepidemiology, 23, 159-169 (2004).

43) Matsuzaki K., Miyazaki K., Sakai S., Yawo H., Nakata N., Moriguchi S., Fukunaga K., Yokosuka A., Sashida Y., Mimaki Y., Yamakuni T., Ohizumi Y., Eur. J. Pharmacol., 578, $194-200$ (2008).

44) Nakajima A., Yamauni T., Matsuzaki K., Nakata N., Onozuka H., Yokosuka A., Sashida Y., Mimaki Y., Ohizumi Y., J. Pharmacol. Exp. Ther., 321, 784-790 (2007). 